\title{
Erwiderung auf die obenstehende Bemerkung
}

Auf die Bemerkung Hamburgers, zu meiner Arbeit Z. f. A., Bd. 63, im einzelnen einzugehen, halte ich für überflüs,sig. da sie sich auf Nebensächlichkeiten bezieht.

Ich habe in meiner Arbeit vor allem unsere Erfahrungen, die im großen ganzen günstig waren, bekanntgeben wollen und die Indikation, wie sie sich aus dieser und der Erfahrung anderer, die in der Literatur niedergelegt ist, herausschält. Dabei mußte ich folgerichtig auch die unglücklichen Zufälle erwähnen, die Egtermeier und Mock erlebt haben. Im Fall Egtermeier ist das Glaukosan nicht nach Vorschrift eingeträu-felt worden. Deshalb behauptet Hamburger. die Schädigung sei auf die unvorschriftsmäßige Anwendung zurückzuführen. Bewiesen hat er es nicht. Im zweiten Falle Mock ist nach Hamburgers Angabe einmal eine Tonometerschädigung auf-getreten, ohne daß ein Tonometer angewandt wurde. Aus die-sen Gründen bin ich „der”, wie Hamburger schreibt, , ,irre-führenden, um nicht zu sagen, grob falschen Ansicht," daß unter besonders ungünstigen Umständen Hornhautschädigungen vorkommen können.

Der zweite Vorwurf, der mir gemacht wird, ist der, daß wir in zwei Fallen die konservative Behandlung zugunsten operativer Eingriffe aufgegeben und einmal Histamin nicht angewandt haben, ein Mittel, das erst einige Monate später der Öffentlichkeit übergeben wurde. Für die Wahl der Behand-lungsmethode sind uns außer unseren Erfahrungen über die Wirksamkeit noch andere Erwägungen maßgebend, z. B. sozialer und geographischer Natur. Unsere Meinung bezüglicb

Erwiderung auf die obenstehende Bemerkung. $\quad 3 * 3$ der Wirksamkeit der Operativnen ist nicht so absprechend wie die Hamburgers, der mit seiner Verurteilung aller operativen Eingriffe ziemlich vereinzelt clastehen dürfte. Auch hat der Erfolg der Behandlung in unseren Fallen den Nutzen der Operation gezeigt. Da sich Druck, Gesichtsfeld und Visus auf derselben Höhe halten, wie bei der Entlassung aus der Klinik, kann man die Patienten in größeren Zwischenräumen zur Nach-untersuchung bestellen und ihnen stundenlange Eisenbahn-fahrten und beschwerliche Wege zur Eisenbahnstation, die bei der Glaukosanbehandiung i 2 mai würhentlich notwendig sein würden, ersparen.

Es ist bedauerlich, daß durch cliese Diskussion, in derNebensächlichkeiten über Gebühr in den Vordergrund gescho-ben werden, der Eindruck erweckt werden kann, als ob wirinfolge schlechter Erfahrungen die Glaukosanbehandiung ab-lehnen, was, wie jeder, der meine Arbeit genau liest, zugebenmuß, nicht zutrifft. Ich giaube, daß der Sache mehr gedientist, wenn man die Nachteile einer Methode zu kennen und zuvermeiden sucht, als wenn man sie durch kritiklose Anwendungin Mißkredit bringt. $\quad$ Alice Gredsted. Ophthalmologische Gesellschaît in Wien.

I. Oräentliche Jahresversammlung vom 16. Januar 1928. A dm ¿nistrativer Teil: Prof. Lindner: Nachruf für Hofrat Primarius 0. Purtscher. Die Neuwahl des Vorstandes ergab: Vorsitzender: Hofrat Prof. Dr. E. Fuchs, Vorsitzender-Stellvertreter: Prof. Dr. K. Lindner, Schrift-führer: Dr. Wm. Kreibig, Säckelwart: Primarius Dr. E. Guzmann.

Wissenschaftlicher Teil : Demonstrationen : 1. Proksch: $\mathrm{K}<>$ ntaktí $>$ läser bei Maculae eorneae. 
Vor kurzem sind in der Ophthalmologisehen Gesellschaft Patienten mit Kontaktgläsern bei Keratokonus demonstriert worden.

B. Bei einer Patientin mit jahrelang bestehendem ekz. Pannus

Visus V24 am besseren Auge

waren verschiedene Mittel - auch abrasio - ohne Erfolg angewendet worden

die Einsetzung eines Kontaktglases besserte den Visus auf 6/3(i. Die Patientin trägt das Glas

täglich über acht Stunden und ist sehr zufrieden.

Verwendet wurden ausnahmslos Kontaktgläser von Zeiss. Bei zwei weiteren Patientinen wurde der Visus deutlich gebessert. Eine davon, 